\title{
CHARACTERIZATION OF CHEMICAL CONSTITUENTS OF Citrullus colocynthis (L.) EXTRACTS AND THEIR CHECKED RELATION TO TOXICITY AGAINST COWPEA APHID, Aphis Craccivora Koch. Heba Y. E. Ibrahim Plant protection Research Institute, ARC, Dokki, Giza, Egypt.
}

\begin{abstract}
Citrullus colocynthis (L.) fruits were separately extracted with different organic solvents (petroleum ether, ethyl acetate and methanol). The volatile components of each solvent extract were characterized and identified by GC/MS technique. The insecticidal properties of each extracts were tested against adults and nymphs of cowpea aphid, Aphis craccivora Koch. Methanol extract was the most efficient against both adults and nymphs with $\mathrm{LC}_{50}$ : $639.68 \mathrm{ppm}$ and $\mathrm{LC}_{50}: 555.9 \mathrm{ppm}$, respectively. Ethyl acetate extract showed moderate efficacy against adults and nymphs with $\mathrm{LC}_{50}: 968.85 \mathrm{ppm}$ and $\mathrm{LC}_{50}: 833.58 \mathrm{ppm}$, respectively. Petroleum ether extract showed weak toxicity against adults and nymphs with $3144.99 \mathrm{ppm}$ and LC $_{50}$ : $2768.52 \mathrm{ppm}$, respectively.
\end{abstract}

Keywords: Aphis craccivora, Citrullus colocynthis, GC/MS analysis of colothyn extract.

\section{INTRODUCTION}

Cowpea Aphid, Aphis craccivora Koch. is a serious pest attacking a wide spectrum of economic plants causing great loss in yield. It infests many legumes, cotton, and as well as Shephered'- purse, lambsquarters, lettuce, pepperweed, Polygonum sp. and Rumex sp. Cowpea aphid transmits nearly 30 plant viruses including cotton curliness virus (Kennedy et al., 1962; Blackman and Eastop, 1984 ). Also, it transmits Peanut stripe virus (PStV) and Peanut mottle virus (PMV) (Sreenivasulu and Demeski, 1988), Chili veinal mottle virus and pepper mottle virus (Cerkauskas, 2004). Moreover, cowpea aphid secretes toxic saliva into the host plants causing symptoms ranged from simple stippling of the plant leaves to extensive disruption of the entire plant (Summers et al., 1996).

The extensive use of traditional insecticides have created many problems, so, the scientists and environmental policy makers seek less toxic alternatives for controlling insect pests. Natural products of plant origin is one of the most important safer alternatives. Bitter apple, Citrullus colocynthis (L.) (Cucurbitaceae) or colothyn has gained deserving attention as natural insecticide because it has deterrent, antifeedant, growth regulating and fertility-reducing properties on insects (Prabuseenivasan, et al., 2004; Pravin et al., 2013 and Soam et al., 2013). Several active chemical constituents of this plant were isolated and characterized. These including bitter substances ( colocynthin and colocynthetin); cucurbitacins A, B, C, D and E ( $\alpha$-elaterin) (Adam et al., 2001); cucurbitacin glycosides (Hatam, et al., 1989 and Abbas, et al., 2006); flavenoides and flavon glycosides (Maatooq, et al., 1997 and 
Abbas et al., 2006) The goal of this study was to characterize the main volatile constituents of different organic solvent extracts of $C$. colocynthis fruit, then, evaluate the insecticidal effects of these extracts against $A$. craccivora in the laboratory.

\section{MATERIALS AND METHODS}

\section{Rearing of cowpea aphid, $A$. craccivora:}

The strain of $A$. craccivora was obtained from the farm of Faculty of Agriculture, Mansoura University, and had been known to be free from insecticidal contamination. Aphid strain was reared on broad bean (2-3 weeks old) planted in small pots $(15 \mathrm{~cm} 3)$ and kept under plastic greenhouse conditions of $25 \pm 5^{\circ} \mathrm{C}, 70 \pm 5 \mathrm{RH}$ and 12:12 L: D. Plants were changed as needed once or twice per week. The transfer of aphids from old plants to new ones was carried out by allowing aphids to over voluntarily from detached leaves placed on new plants or by artist s brush.

\section{Extraction of the active fractions from tested plant:}

Fruits of $C$. colocynthis were grinding into fine powder by using electric mill then it was weighted and apportioned to three equal portions. The three portions were separately soaked in Petrolleum ether, ethyl acetate and methanol for about a week then well shacked and filtered, then, washed by the same solvent three times and let solvents evaporate using rotator evaporator. The extracted crudes were kept in deep freezer until use.

\section{Chemical study:}

A sample of each $C$. colocynthis extract was analyzed by GC/MS technique for characterization and identification of its volatile components. GC/MS analysis of the volatile fractions were performed on a Varian GC interfaced to Finnegan SSQ 7000 Mass selective Detector (SMD) with ICIS V2.0 data system for MS identification of the GC components. The column used was DB-5 ( $\mathrm{J} \& \mathrm{~W}$ Scientific, Folosm, CA) cross-linked fused silica capillary column ( $30 \mathrm{~m}$. long, $0.25 \mathrm{~mm}$. internal diameter) coated with poly dimethyl-siloxane $(0.5 \mu \mathrm{m}$. film thickness). The oven temperature was programmed from $50 \mathrm{oC}$ for $3 \mathrm{~min}$., at isothermal, then heating by $7 \mathrm{oC} / \mathrm{min}$. to $250 \mathrm{oC}$ and isothermally for $10 \mathrm{~min}$., at $2500 \mathrm{C}$.Injector temperature was $200 \mathrm{oC}$ and the volume injected was $0.5 \mu \mathrm{l}$. Transition-line and ion source temp. were $250 \mathrm{oC}$ and $150 \mathrm{oC}$, respectively. The mass spectrometer had a delay of $3 \mathrm{~min}$. to avoid the solvent plead and then scanned from $\mathrm{m} / \mathrm{z} 50$ to 300. Ionization energy was set at $70 \mathrm{eV}$. (Faculty of Pharmacy, Mansoura Univ.).

Bioassay study:

Plant leaves were transferred to $15 \mathrm{~cm}$ Petri - dishes. Each ten aphid individuals of the same age were transferred to a Petri-dish to be considered as one replicate. Each concentration had three replicates and another three replicates sprayed only with water and $0.05 \%$ aqueous Tween 80 to be considered as control. In case of testing the efficiency on nymphal stage, the adults were allowed to lay nymphs on the surface of the host leaves for a period of 24 hours, then the parents were removed and nymphs were treated 
when reaching the age of two days. Cowpea aphid had been treated with different concentrations of extracts by spraying methods, then the lids of Petri-dishes were sealed and placed in an incubator at $25 \pm 2 \mathrm{C}^{\circ}, 70 \pm 5 \%$ $\mathrm{RH}$., and photoperiod 12:12 hs L:D. Observations were recorded daily and the experiment continued for seven days. Broad bean leaves were replaced by fresh ones after first three days of the treatment to provide a source of nutrition.

At the end of this period, the average of mortality percentages of $A$. craccivora was estimated and corrected using Abbott's formula (1925). The corrected mortality percentage of each extract was statistically calculated according to Finney (1971). The corresponding concentration probit lines (LC-p lines) were estimated in addition to determination of $\mathrm{LC}_{50}, \mathrm{LC} 90$, slope values and toxicity index according to Sun`s equation.

\section{RESULTS AND DISCUSSION}

\section{Chemical study:}

The GC/MS chromatogram showed sixteen peaks corresponding to sixteen compounds. These compounds were characterized by comparing their mass spectra with those of their analogous reported by NIST library. The obtained results were reported in Table (1). Compound 12, $(9,12-$ Octadecadienoic acid) was found to be antiinflammatory, hypocholesterolemic, cancer preventive, hepatoprotective, nematicide, insectifuge, antihistaminic, antieczemic, antiacne, 5-alpha reductase inhibitor, antiandrogenic, antiarthritic and anticoronary. (Maruthupandian and Mohan, 2011). Also, it was reported that compound 14, (oleic acid) has insecticidal, herbicidal and fungicida activities. 
Heba Y. E. Ibrahim

Table (1): The GC/MS an analysis of Pet. ether fraction of C. colocynthis

\begin{tabular}{|c|c|c|c|c|c|}
\hline S.N & Compound name & R.T. & Area \% & M.F. & Compound Structure \\
\hline 1 & Nonane & 4.39 & 0.85 & $\mathrm{C}_{9} \mathrm{H}_{20}$ & \\
\hline 2 & Decane & 6.41 & 1.44 & $\mathrm{C}_{10} \mathrm{H}_{22}$ & \\
\hline 3 & Undecane & 8.14 & 1.61 & $\mathrm{C}_{11} \mathrm{H}_{24}$ & \\
\hline 4 & Dodecane. & 9.67 & 1.23 & $\mathrm{C}_{12} \mathrm{H}_{26}$ & \\
\hline 5 & 9-Hexadecenoic acid & 14.24 & 0.50 & $\mathrm{C}_{16} \mathrm{H}_{30} \mathrm{O}_{2}$ & \\
\hline 6 & Tridecane. & 14.79 & 2.84 & $\mathrm{C}_{13} \mathrm{H}_{28}$ & \\
\hline 7 & Hexadecane. & 14.79 & 2.84 & $\mathrm{C}_{16} \mathrm{H}_{34}$ & \\
\hline 8 & Tetradecane. & 14.79 & 2.84 & $\mathrm{C}_{14} \mathrm{H}_{30}$ & \\
\hline 9 & Nondecane. & 16.96 & 3.80 & $\mathrm{C}_{19} \mathrm{H}_{40}$ & \\
\hline 10 & Pentadecane. & 17.96 & 1.84 & $\mathrm{C}_{15} \mathrm{H}_{32}$ & \\
\hline 11 & n- Hexadecanoic acid. & 18.63 & 1.44 & $\mathrm{C}_{16} \mathrm{H}_{32} \mathrm{O}_{2}$ & \\
\hline 12 & $\begin{array}{c}\text { 9,12-Octadecadienoic } \\
\text { acid. }\end{array}$ & 20.20 & 2.45 & $\mathrm{C}_{18} \mathrm{H}_{32} \mathrm{O}_{2}$ & \\
\hline 13 & Linoleic acid ethyl ester. & 20.20 & 2.45 & $\mathrm{C}_{20} \mathrm{H}_{36} \mathrm{O}_{2}$ & $\pi$ \\
\hline 14 & Oleic Acid. & 20.51 & 1.84 & $\mathrm{C}_{18} \mathrm{H}_{34} \mathrm{O}_{2}$ & \\
\hline 15 & $\begin{array}{c}\text { Hexanedioic acid, dioctyl } \\
\text { ester. }\end{array}$ & 21.32 & 0.44 & $\mathrm{C}_{22} \mathrm{H}_{42} \mathrm{O}_{4}$ & \\
\hline 16 & Geranylgeraniol. & 28.67 & 7.94 & $\mathrm{C}_{20} \mathrm{H}_{34} \mathrm{O}$ & \\
\hline
\end{tabular}




\section{Study of ethyl acetate fraction:}

The GC/MS chromatogram showed fifty peaks corresponding to fifty compounds. These compounds were characterized by comparing their mass spectra with those of their analogous reported by NIST library. The obtained results were reported in Table| (2). Compound 23, (9,12-octadecadienoic acid, methyl ester) was already isolated from $C$. colocynthis fruits and Mentha microphylla by Farghaly et al. (2009) who proved the toxicity of it against whitefly (Bemisia tabaci) and aphid (A. craccivora). Also, Antonious et al., (2007), proved the toxicity of this compound as insecticide against cabbage hooper, Trichopulsia ni larvae.

Table ( 2 ): The GC/MS analysis of ethyl acetate fraction of $C$. colocynthis

\begin{tabular}{|c|c|c|c|c|c|}
\hline S.N & Compound No. & R.T. & Area $\%$ & M.F. & Compound Structure \\
\hline 1 & $\begin{array}{l}\text { 2-Pentanone, 4-hydroxy-4- } \\
\text { methyl- }\end{array}$ & 3.54 & 35.75 & $\mathrm{C}_{6} \mathrm{H}_{12} \mathrm{O}_{2}$ & 1 \\
\hline 2 & $\begin{array}{c}\text { 13-Docosenoic acid, methyl } \\
\text { ester. }\end{array}$ & 4.06 & 0.18 & $\mathrm{C}_{23} \mathrm{H}_{44} \mathrm{O}_{2}$ & \\
\hline 3 & $\begin{array}{l}\text { 1-Butanol, 3-methyl-, } \\
\text { acetate }\end{array}$ & 4.06 & 0.18 & $\mathrm{C}_{7} \mathrm{H}_{14} \mathrm{O}_{2}$ & \\
\hline 4 & Digitoxin & 10.72 & 0.03 & $\mathrm{C}_{41} \mathrm{H}_{64} \mathrm{O}_{13}$ & \\
\hline 5 & $\begin{array}{c}\text { 6-(p-Tolyl)-2-methyl-2- } \\
\text { heptenol }\end{array}$ & 13.33 & 0.18 & $\mathrm{C}_{15} \mathrm{H}_{22} \mathrm{O}$ & \\
\hline 6 & $\begin{array}{c}\text { 1,3,6,10-Dodecatetraene, } \\
\text { 3,7,11-trimethyl -, (Z,E)- }\end{array}$ & 13.48 & 0.29 & $\mathrm{C}_{15} \mathrm{H}_{24}$ & \\
\hline 7 & Pterin-6-carboxylic acid & 13.48 & 0.29 & $\mathrm{C}_{7} \mathrm{H}_{5} \mathrm{~N}_{5} \mathrm{O}_{3}$ & \\
\hline 8 & Zingiberene & 14.19 & 0.13 & $\mathrm{C}_{15} \mathrm{H}_{24}$ & \\
\hline 9 & Guanosine & 14.64 & 0.15 & $\mathrm{C}_{10} \mathrm{H}_{13} \mathrm{~N}_{5} \mathrm{O}_{5}$ & \\
\hline
\end{tabular}


Heba Y. E. Ibrahim

Continue Table (2): The GC/MS analysis of ethyl acetate fraction of $C$. colocynthis

\begin{tabular}{|c|c|c|c|c|c|}
\hline S.N & Compound No. & R.T. & Area $\%$ & M.F. & Compound Structure \\
\hline 10 & $\begin{array}{l}\text { d-Glycero-d-galacto- } \\
\text { heptose }\end{array}$ & 14.64 & 0.15 & $\mathrm{C}_{7} \mathrm{H}_{14} \mathrm{O}_{7}$ & \\
\hline 11 & Arginine & 15.78 & 0.07 & $\mathrm{C}_{6} \mathrm{H}_{14} \mathrm{~N}_{4} \mathrm{O}_{2}$ & \\
\hline 12 & $\begin{array}{l}\text { 2-Pentadecanone, } 6,10,14- \\
\text { trimethyl- }\end{array}$ & 17.30 & 0.05 & $\mathrm{C}_{18} \mathrm{H}_{36} \mathrm{O}$ & \\
\hline 13 & Folic Acid & 17.85 & 0.09 & $\mathrm{C}_{19} \mathrm{H}_{19} \mathrm{~N}_{7} \mathrm{O}_{6}$ & \\
\hline 14 & $\begin{array}{l}\text { Cyclopentaneundecanoic } \\
\text { acid, methyl ester }\end{array}$ & 18.11 & 0.6 & $\mathrm{C}_{17} \mathrm{H}_{32} \mathrm{O}_{2}$ & \\
\hline 15 & $\begin{array}{c}\text { Tridecanoic acid, methyl } \\
\text { ester }\end{array}$ & 18.11 & 0.6 & $\mathrm{C}_{14} \mathrm{H}_{28} \mathrm{O}_{2}$ & \\
\hline 16 & Methyl tetradecanoate & 18.11 & 0.6 & $\mathrm{C}_{15} \mathrm{H}_{30} \mathrm{O}_{2}$ & \\
\hline 17 & n-Hexadecanoic acid & 18.50 & 1.48 & $\mathrm{C}_{16} \mathrm{H}_{32} \mathrm{O}_{2}$ & \\
\hline 18 & Undecanoic acid (35) & 18.50 & 1.48 & $\mathrm{C}_{11} \mathrm{H}_{22} \mathrm{O}_{2}$ & \\
\hline 20 & $\begin{array}{c}\text { Hexadecanoic acid, ethyl } \\
\text { ester }\end{array}$ & 18.76 & 0.28 & $\mathrm{C}_{18} \mathrm{H}_{36} \mathrm{O}_{2}$ & \\
\hline 21 & $\begin{array}{l}\text { Dodecanoic acid, 3- } \\
\text { hydroxy- }\end{array}$ & 18.76 & 0.28 & $\mathrm{C}_{12} \mathrm{H}_{24} \mathrm{O}_{3}$ & \\
\hline 22 & Oleic Acid & 18.76 & 0.28 & $\mathrm{C}_{18} \mathrm{H}_{34} \mathrm{O}_{2}$ & \\
\hline
\end{tabular}


J. Plant Prot. and Path., Mansoura Univ., Vol.6 (10), october, 2015

Continue Table (2 ): The GC/MS analysis of ethyl acetate fraction of $C$. colocynthis

\begin{tabular}{|c|c|c|c|c|c|}
\hline S.N & Compound No. & R.T. & Area\% & M.F. & Compound Structure \\
\hline 23 & $\begin{array}{c}\text { 9,12-Octadecadienoic acid, } \\
\text { methyl ester. }\end{array}$ & 19.69 & 2.51 & $\mathrm{C}_{19} \mathrm{H}_{34} \mathrm{O}_{2}$ & \\
\hline 24 & $\begin{array}{l}\text { Hexadecanoic acid, 15- } \\
\text { methyl-, methyl ester. }\end{array}$ & 19.97 & 0.31 & $\mathrm{C}_{17} \mathrm{H}_{32} \mathrm{O}_{2}$ & \\
\hline 25 & Decanoic acid, methyl ester & 19.97 & 0.31 & $\mathrm{C}_{11} \mathrm{H}_{22} \mathrm{O}_{2}$ & \\
\hline 26 & $\begin{array}{c}\text { Heneicosanoic acid, methyl } \\
\text { ester }\end{array}$ & 19.97 & 0.31 & $\mathrm{C}_{22} \mathrm{H}_{44} \mathrm{O}_{2}$ & \\
\hline 27 & 9,12-Octadecadienal & 20.09 & 2.66 & $\mathrm{C}_{18} \mathrm{H}_{32} \mathrm{O}$ & \\
\hline 28 & $\begin{array}{l}\text { 9,12-Octadecadienoyl } \\
\text { chloride, }(Z, Z)-\end{array}$ & 21.90 & 0.26 & $\mathrm{C}_{18} \mathrm{H}_{31} \mathrm{ClO}$ & \\
\hline 29 & Agaricic acid & 23.18 & 0.08 & $\mathrm{C}_{22} \mathrm{H}_{40} \mathrm{O}_{7}$ & \\
\hline 30 & Scilliroside & 23.21 & 0.07 & $\mathrm{C}_{32} \mathrm{H}_{44} \mathrm{O}_{2}$ & \\
\hline 31 & Paromomycin I & 24.25 & 0.08 & $\mathrm{C}_{23} \mathrm{H}_{45} \mathrm{~N}_{5} \mathrm{O}_{14}$ & \\
\hline 32 & $\begin{array}{l}\text { Tetradecane, 2,6,10- } \\
\text { trimethyl- }\end{array}$ & 25.06 & 5.86 & $\mathrm{C}_{17} \mathrm{H}_{36}$ & \\
\hline 33 & Octadecane, 1-(ethenyloxy)- & 25.24 & 3.23 & $\mathrm{C}_{20} \mathrm{H}_{40} \mathrm{O}$ & \\
\hline 34 & $\begin{array}{c}\text { 2,6,10-Dodecatrien-1-ol, } \\
\text { 3,7,11-trimethyl- }\end{array}$ & 25.42 & 2.52 & $\mathrm{C}_{15} \mathrm{H}_{26} \mathrm{O}$ & \\
\hline 35 & $\begin{array}{c}\text { 9,10-Secocholesta- } \\
\text { 5,7,10(19)-triene-3,24,25- } \\
\text { triol, (3á,5Z,7E)- }\end{array}$ & 26.91 & 0.43 & $\mathrm{C}_{27} \mathrm{H}_{44} \mathrm{O}_{3}$ & \\
\hline
\end{tabular}


Heba Y. E. Ibrahim

Continue Table (2 ): The GC/MS analysis of ethyl acetate fraction of $C$. colocynthis

\begin{tabular}{|c|c|c|c|c|c|}
\hline S.N & Compound No. & R.T. & Area\% & M.F. & Compound Structure \\
\hline 36 & Gibberellic acid & 26.91 & 0.43 & $\mathrm{C}_{19} \mathrm{H}_{22} \mathrm{O}_{6}$ & \\
\hline 37 & $\begin{array}{l}\text { Pregn-5-ene- } \\
\text { 3,8,11,12,14,20-hexol, } \\
\text { (3á,11à,12á,14á)- }\end{array}$ & 27.27 & 0.03 & $\mathrm{C}_{21} \mathrm{H}_{34} \mathrm{O}_{6}$ & \\
\hline 38 & $\begin{array}{c}\text { 1,6,10,14-Hexadecatetraen- } \\
\text { 3-ol, 3,7,11,15-tetramethyl-, } \\
-(E, E)-\end{array}$ & 28.56 & 4.09 & $\mathrm{C}_{20} \mathrm{H}_{34} \mathrm{O}$ & \\
\hline 39 & Geranylgeraniol & 28.56 & 4.09 & $\mathrm{C}_{20} \mathrm{H}_{34} \mathrm{O}$ & \\
\hline 40 & $\begin{array}{l}\text { 10-Heptadecen-8-ynoic } \\
\text { acid, methyl ester, (E)- }\end{array}$ & 28.83 & 1.84 & $\mathrm{C}_{18} \mathrm{H}_{30} \mathrm{O}_{2}$ & \\
\hline 41 & Nitro-L-arginine & 30.05 & 0.07 & $\mathrm{C}_{6} \mathrm{H}_{13} \mathrm{~N}_{5} \mathrm{O}_{4}$ & \\
\hline 42 & $\begin{array}{c}\text { Olean-12-ene- } \\
\text { 3,15,16,21,22,28-hexol, } \\
\text { (3á,15à,16à,21á,22à)- }\end{array}$ & 30.19 & 0.34 & $\mathrm{C}_{30} \mathrm{H}_{50} \mathrm{O}_{6}$ & \\
\hline 43 & Glucobrassicin & 30.19 & 0.34 & $\mathrm{C}_{10} \mathrm{H}_{9} \mathrm{~N}_{2} \mathrm{~S}_{2} \mathrm{O}_{4}$ & \\
\hline 44 & Dasycarpidan-1-one & 30.41 & 0.04 & $\mathrm{C}_{17} \mathrm{H}_{20} \mathrm{~N}_{2} \mathrm{O}$ & \\
\hline 45 & Vitamin A palmitate & 30.41 & 0.04 & $\mathrm{C}_{36} \mathrm{H}_{60} \mathrm{O}_{2}$ & \\
\hline 46 & Cholic acid & 32.04 & 0.69 & $\mathrm{C}_{24} \mathrm{H}_{40} \mathrm{O}_{5}$ & \\
\hline
\end{tabular}


Continue Table (2): The GC/MS analysis of ethyl acetate fraction of $C$. colocynthis

\begin{tabular}{|l|c|c|c|c|c|}
\hline S.N & Compound No. & R.T. & Area\% & M.F. & Compound Structure \\
\hline 47 & $\begin{array}{c}\text { psi.,.psi.-Carotene, 1,1',2,2'- } \\
\text { tetrahydro-1,1'-dimethoxy- }\end{array}$ & 32.39 & 0.29 & $\mathrm{C}_{42} \mathrm{H}_{64} \mathrm{O}_{2}$ & (1) \\
\hline 48 & $\begin{array}{c}\text { Hydrocortisone } \\
\text { hemisuccinate [anhydrous] }\end{array}$ & 36.65 & 0.37 & $\mathrm{C}_{25} \mathrm{H}_{34} \mathrm{O}_{8}$ & \\
\hline 49 & $\begin{array}{c}\text { Picrotoxinin } \\
50\end{array}$ & 32.88 & 0.06 & $\mathrm{C}_{15} \mathrm{H}_{16} \mathrm{O}_{6}$ & \\
\hline $\begin{array}{c}\text { Corynan-17-ol, 19- } \\
\text { didehydro-10-methoxy- }\end{array}$ & 38.76 & 0.1 & $\mathrm{C}_{7} \mathrm{H}_{8} \mathrm{NO}$ & \\
\hline
\end{tabular}

\section{Study of methanol fraction:}

The GC/MS chromatogram showed twenty peaks corresponding to twenty compounds. These compounds were characterized by comparing their mass spectra with those of their analogous reported by NIST library. The obtained results were reported in Table (3).

Table (3): The GC/MS analysis of Methanol fraction of C. colocynthis

\begin{tabular}{|l|c|c|c|c|c|}
\hline S.N & Compound No. & R.T. & Area\% & M.F. & Compound Structure \\
\hline 1 & Cystine & 2.70 & 0.86 & $\mathrm{C}_{6} \mathrm{H}_{12} \mathrm{~N}_{2} \mathrm{O}_{4} \mathrm{~S}_{2}$ & \\
\hline 2 & Acetaldehyde & 2.70 & 0.86 & $\mathrm{C}_{2} \mathrm{H}_{4} \mathrm{O}$ & \\
\hline 3 & Undecane & 8.00 & 1.34 & $\mathrm{C}_{11} \mathrm{H}_{24}$ & \\
\hline 4 & Pterin-6- arboxylic acid & 12.05 & 2.37 & $\mathrm{C}_{7} \mathrm{H}_{5} \mathrm{~N}_{5} \mathrm{O}_{3}$ & \\
\hline 5 & Oleic Acid & 18.32 & 2.13 & $\mathrm{C}_{18} \mathrm{H}_{34} \mathrm{O}_{2}$ &
\end{tabular}


Heba Y. E. Ibrahim

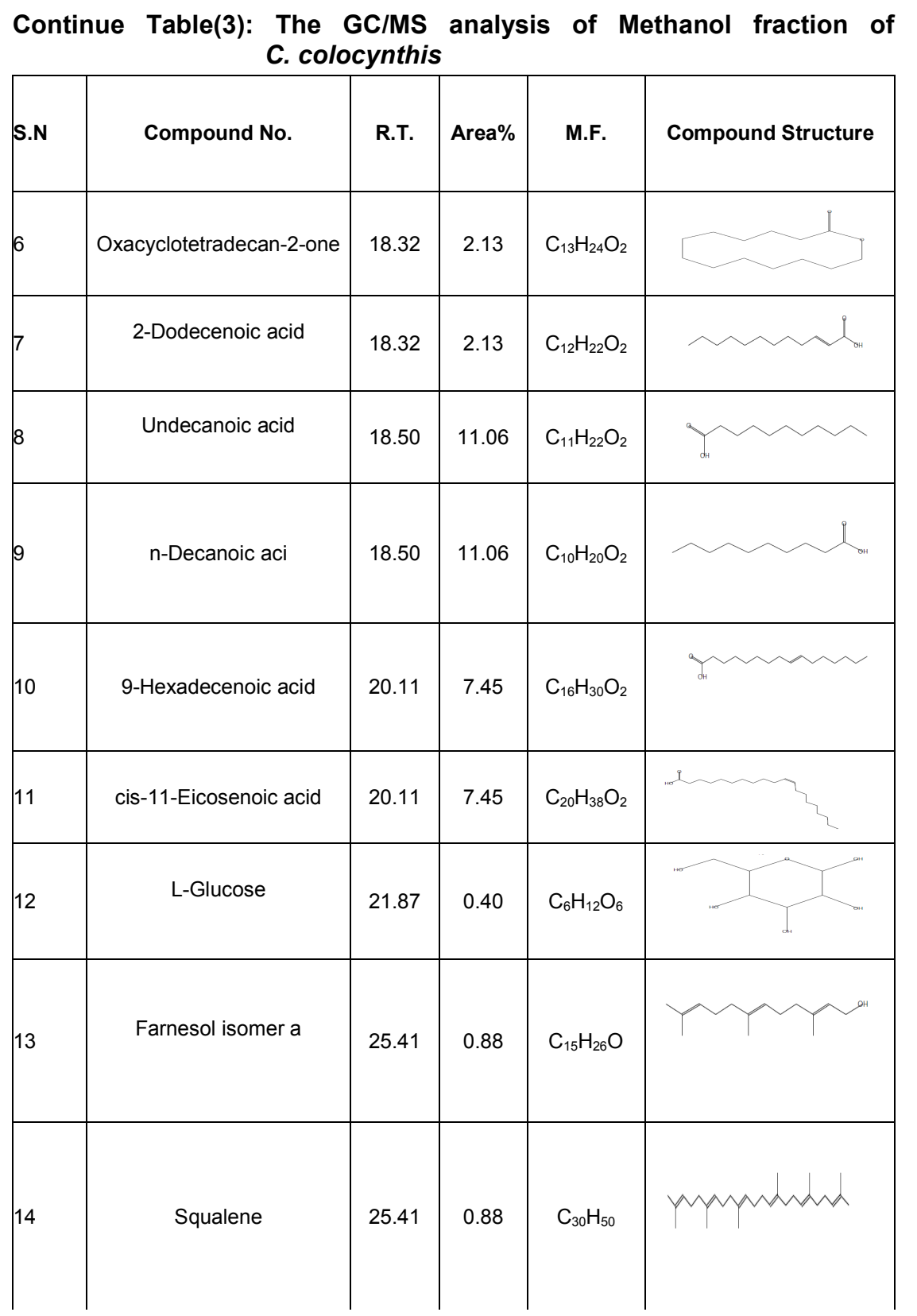


Continue Table (3) : The GC/MS analysis of Methanol fraction of C. colocynthis

\begin{tabular}{|c|c|c|c|c|c|}
\hline S.N & Compound No. & R.T. & Area $\%$ & M.F. & Compound Structure \\
\hline 15 & Calcitriol & 27.33 & 0.67 & $\mathrm{C}_{27} \mathrm{H}_{44} \mathrm{O}_{3}$ & \\
\hline 16 & Picrotoxin & 27.33 & 0.67 & $\mathrm{C}_{30} \mathrm{H}_{34} \mathrm{O}_{13}$ & \\
\hline 17 & Aldosterone & 27.79 & 0.25 & $\mathrm{C}_{21} \mathrm{H}_{28} \mathrm{O}_{5}$ & \\
\hline 18 & Caryophyllene oxide & 28.56 & 6.05 & $\mathrm{C}_{15} \mathrm{H}_{24} \mathrm{O}$ & \\
\hline 19 & $\begin{array}{c}\text { 9,10- ecocholesta- } \\
\text { 5,7,10(19)-triene-3,24, 25- } \\
\text { triol, (3á,5Z,7E)- }\end{array}$ & 28.84 & 4.64 & $\mathrm{C}_{27} \mathrm{H}_{44} \mathrm{O}_{3}$ & \\
\hline 20 & $\begin{array}{c}\text { 3,4-Dimethoxycinnam-ic } \\
\text { acid }\end{array}$ & 29.88 & 5.04 & $\mathrm{C}_{11} \mathrm{H}_{12} \mathrm{O}_{4}$ & \\
\hline
\end{tabular}

\section{Bioassay Study:}

Efficiency of different solvent extracts of colothyn extracts against $A$. craccivora:

Data in Tables (4\&5) showed the efficiency of colothyn extracts against both of adults and nymphs of cowpea aphid. Data cleared that the mortality percent of both of adults and nymphs increased with increasing the tested extract concentrations. Methanol extract was the most effective one against both adults and nymphs, followed by ethyl acetate extract that showed moderate efficiency and then petroleum ether extract. For adults, Methanol extract showed $\mathrm{LC}_{50}$ of $639.68 \mathrm{ppm}$ and $\mathrm{LC}_{90}$ of the $2472.4 \mathrm{ppm}$ followed by ethyl acetate extract with $\mathrm{LC}_{50}$ of $968.85 \mathrm{ppm}$ and $\mathrm{LC}_{90}$ of $5429.94 \mathrm{ppm}$, then 
petroleum ether extract with $L C_{50}$ of $3144.99 \mathrm{ppm}$ and $L C_{90}$ of $32620.61 \mathrm{ppm}$. Regarding to nymphs, they showed more susceptibility to all extracts. Methanol extract was the most effective with $\mathrm{LC}_{50}$ of $555.9 \mathrm{ppm}$ and $\mathrm{LC}_{90}$ of $2580.28 \mathrm{ppm}$, followed by ethyl acetate extract with $\mathrm{LC}_{50}$ of $833.58 \mathrm{ppm}$ and $\mathrm{LC}_{90}$ of $4325.47 \mathrm{ppm}$, then petroleum ether extract with $\mathrm{LC}_{50}$ of $2768.52 \mathrm{ppm}$ and $\mathrm{LC}_{90}$ of $27195.44 \mathrm{ppm}$.

Table 4: Efficiency of colothyn extracts against adults of A.craccivora under laboratory conditions of $25 \pm 2 \mathrm{C}^{0}$ and $70 \pm 5 \% \mathrm{RH}$.

\begin{tabular}{|l|c|c|c|c|}
\hline Treatment & Slope & LC $_{\mathbf{5 0}}$ & $\mathbf{L C}_{\mathbf{9 0}}$ & Toxicity index \\
\hline Petroleum ether extract & 1.26 & 3144.99 & 32620.61 & 20.34 \\
\hline Ethyl acetate extract & 1.71 & 968.85 & 5429.94 & 66.02 \\
\hline Methanol extract & 2.18 & 639.68 & 2472.4 & 100 \\
\hline
\end{tabular}

Table 5: Efficiency of colothyn extracts against nymphs of A.craccivora under laboratory conditions of $25 \pm 2 \mathrm{C}^{0}$ and $70 \pm 5 \% \mathrm{RH}$.

\begin{tabular}{|l|c|c|c|c|}
\hline Treatment & Slope & LC $_{50}$ & $\mathbf{L C}_{90}$ & Toxicity index \\
\hline Petroleum ether extract & 1.29 & 2768.52 & 27195.44 & 20.08 \\
\hline Ethyl acetate extract & 1.79 & 833.58 & 4325.47 & 66.69 \\
\hline Methanol extract & 1.92 & 555.9 & 2580.28 & 100 \\
\hline
\end{tabular}

The differences in chemical constituents of different solvent extracts of C. colocynthis fruits may explain the observed differences of efficacy of the three different extracts. Results obtained about colothyn extracts toxicity to cowpea aphid agreed with Farghaly et al. (2009), who revealed that the methanol extract of colothyn was the most effective with $\mathrm{LC}_{50}$ of $621.94 \mathrm{ppm}$. Also, agreed with Torkey et al. (2009) who evaluated the toxicity of different solvent extracts of $C$. colocynthis fruit against $A$. craccivora. He revealed that the highest insecticidal effect was obtained from ethanol extract.

\section{REFERENCES}

Abbas, D.; Simon, G.; Ali, R.; Hossein, N.; Masoud, M.; Lutfan, N. and Satyajtt, D. (2006). Flavone C-glycoside and cucurbitacin glycoside from Citrullus colocynthis. DARA, 14(3): 109-114.

Abbott, W. S. (1925). A method for computing the effectiveness an insecticide. J. Econ. Entomol., 18: 265-267.

Adam, S.; Al-Yahya, M. and Al-Farhan, A. (2001) Response of Najdi sheep to oral administration of Citrullus colocynthis fruits. Small Rumin. Res., 40: 239- 244.

Antonious, G. F.; Meyer, J. E.; Rogers, J. A. and Yoon-Hyeon Hu (2007) Growing hot pepper for cabbage looper, Trichopulsia ni (Hübner) and spider mite, Tetranychus urticae (Koch.) control. J. of Environ.Sci. and Health. Part B., 42: 559- 567.

Blackman, R. L. and Eastop, V. F. (1984). " Aphids on the world's crops: An identification and information Guide". Wiley, New York.

Cerkauskas (2004). AVRDC-The World Vegetable Center, Fac. Sheet, htt://www. Avrdc. Org. 
Farghaly, S. F.; Torkey, H. M. and Abou-Yousef, H. A. (2009) Natural extracts and their chemical constituents in relation to toxicity against whitefly (Bemicia tabaci) and aphid (Aphis craccivora). Australian J. of Basic and Applied Sci., 3(4): 3217- 3223.

Finney, D. J. (1971). Probit analysis. A Statistical Treatment of the Sigmoid Response Curve. 7th Ed., Cambridge Univ. Press, England.

Hatam, N. A.; Whiting, D. A. and Yousif, N. J. (1989) Cucurbitacin glycosides from Citrullus colocynthis. Phytochemistry, 28: 1268- 1271.

Kennedy, J. S.; Day, M. F. and Eastop, V. F. (1962). A conceptus of aphids as vectors of plant viruses. Commonwealth Institute of Ent., London, $114 \mathrm{pp}$.

Maatooq, G.; El-Sharkawy, S.; Afifi, M and Rosazza, P. (1997) C- $\rho-$ Hydroxybenzoyl-glycoflavanones from Citrullus colocynthis. Phytochemistry, 44: 187- 190.

Maruthupandian, A. and Mohan, V. R. (2011). GC-MS analysis of some bioactive constituents of Pterocarpus marsupium Roxb. International Journal of Chem. Tech. Research. 3(3): 1652-1657.

Prabuseenivasan, S.; Jayakumar, M.; Raja, N. and Ignacimuthu, S. (2004) Effect of bitter apple, Citrullus colocynthis (L.) Schrad seed extracts against pulse beetle, Callosobruchus maculatus Fab. (Coleoptera: Brachidae). Entomol., 29: 81-84.

Pravin, B.; Tushar, D.; Vijay, P. and Kishanchnad, K. (2013) Review on Citrullus colocynthis. IJRPC. 3(1): 46- 53.

Soam, P. S.; Singh, T. and Vijayvergia, R. (2013) Citrullus colocynthis (LINN.) and Luffa acutangula (L.) roxb, Schrad source of bioinsecticides and their contribution in managing climate change. IJABPT. 4(4): 7-9.

Sreenivasulu, P. and Demeski, J. W. (1988). Transmission of Peanut Mottle and Peanut Stripe viruses by Aphis craccivora and Myzus persicae. Plant disease, The American Phytopathol. Soci., 72: 722-723.

Summers, C. G.; Godfrey, L. D. and Long, R. (1996). Sugar beet: Empoasca leafhopper. UC IPM Pest Management Guidelines. UC IPM Pest Guideline Series, UCDANR Puplication 3339.

Sun, Y. P. (1950). Toxicity index an improved method of comparing the relative toxicity of insecticides. J. Econ. Entomol., 43: 45-53.

Torkey, H. M.; Abou-Yousif, H. M.; Abdel Azeiz, A. Z. and Hoda, E. A. Farid (2009) Insecticidal effect of cucurbitacin E Glycoside isolated from Citrullus colocynthis against Aphis craccivora. Australian J. of Basic and applied Sci., 3(4): 4060- 4066. 
تعريف المكونات الكيميائية لمستخلصات نبات الحنظل و علاقتها بالسمية ضد من اللوبيا.

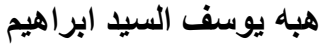
معهز بحوث وقاية النباتات ـ مركز البحوث الزراعية. الدقىـ الجيزة- مصر.

تم استخلاص مكونات ثمار الحنظل باستخدام المذيبات العضوية المختلفة (الايثير البترولي، أسيتات

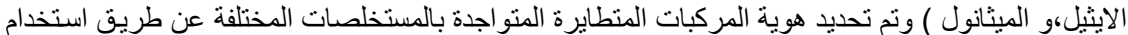

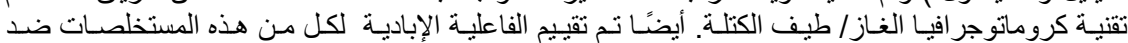

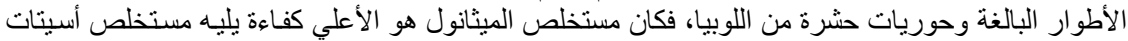

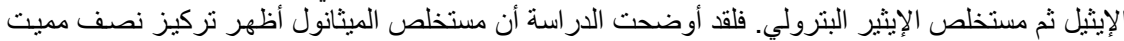

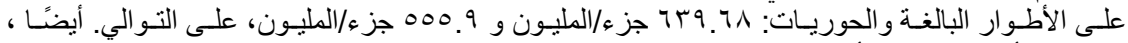

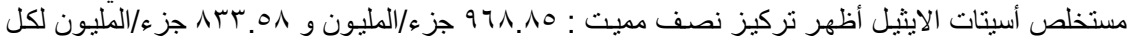

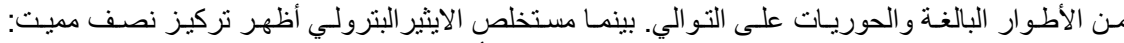

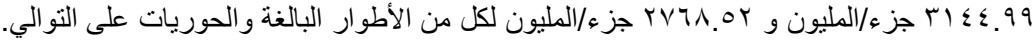

